\title{
Capturing, measuring and responding to changes that influence travel behavior
}

\author{
Khandker M. Nurul Habib ${ }^{1}$ - Stacey G. Bricka ${ }^{2}$
}

Published online: 24 September 2015

(C) Springer Science+Business Media New York 2015

Much of transportation planning centers about change. From the long range planning efforts of evaluating impacts of varying demographic and land use configurations of regional scales to the localized planning efforts of evaluating mode choice variations, understanding and measuring effects of changes on the transportation system performances and travel demands are critical to the profession's ability to meet future transportation needs. This special edition focuses on analyses that detail the behavioral aspects of 'change' through the changes in work status, travel destinations, and service interruptions as well as future changes associated with technology shifts. The special issue also considers the capture and measurement of change, through an evaluation of applications of discount rates, value of time, and a hidden Markov approach.

This Special Issue represents a long-standing collaboration between Transportation and the Transportation Research Board (Standing Committees on Travel Behavior and Values and Demand Forecasting). It presents seven outstanding papers that were originally presented at the 2015 Transportation Research Board Annual Meeting. The papers were selected based on a common theme of 'capturing, measuring and responding to changes that influence travel behaviour' and representations of the state-of-the-art in research as well as potential applications in the real world.

The first four papers explore how changes in specific circumstances lead to changes in travel behavior. The remaining three papers focus on techniques to capture and model changes.

Khandker M. Nurul Habib

khandker.nurulhabib@utoronto.ca

Stacey G. Bricka

sbricka@etcinstitute.com

1 Department of Civil Engineering, University of Toronto, 35 St. George Street, Toronto, ON M5S 1A4, Canada

2 ETC Institute, 725 W. Frontier Circle, Olathe, KS 66061, USA 
The opening paper is "Employment status transitions and shifts in daily activity-travel behavior with special focus on shopping duration" authored by Soora Rasouli, Harry Timmermans and Peter van der Waerden. Authors focused on the change in employment status as an important catalyst of changes in travel behaviour with particular focus on shopping activity duration. Drawing across consecutive waves of Puget Sound Panel Survey data, the authors used descriptive analyses and a random parameters panel effects regression model to illustrate the theory that critical shifts in life events trigger changes in how households and individuals organize their activities. An interesting connection between work status and shopping activity is that work activity generates household income, which is spent in shopping activities. Such an obvious influence was largely overlooked in previous research works. Results reveal an asymmetric relationship between changes in work status and changes in activity travel patterns in general and shopping activity duration in particular.

Pnevmatikou, Karlaftis and Kepaptsoglou co-authored the paper, "Metro Service Disruptions: How Do People Choose to Travel?" the authors combine information on traveler experiences and perceptions in order to model mode choice during a long-run metro service disruption using a nested logit model. This paper is a successful example of application of data fusions for travel behaviour change analysis. Result reveals that flexible work schedule may reduce car dependency during transit service disruption, as well as confirm the influence of income on mode choice. This research informs not only the development of mode choice models but can inform scenario planning for emergency evacuation measures. This paper is devoted to Dr. Karlaftis who was a rising star in the field, but unfortunately passed away in June 2014.

Zeller authored the third paper; "Potential Changes in Transportation Patterns of New York Islanders Fans due to Stadium Relocation." The paper investigates how travel patterns of Hockey fans may be affected by change of the location of a professional hockey team in the New York City region. The study uses a dataset collected through an online survey among the fans of the men's hockey team about their travel patterns toward the current location and expected changes in travel behaviour for the new location. Empirical investigation reveal that fans who are currently using public transit to go to the stadium will most likely to have similar travel patterns despite the change in stadium location. The paper clearly reveals that familiarity with public transit system helps mitigating effects of changes in public event locations on travel behaviour.

The final paper in this first set was co-authored by Vogt, Wang, Gregor and Bettinardi and is titled, "Potential changes to travel behaviors and patterns: a fuzzy cognitive map (FCM) modeling approach." The paper used the FCM approach to investigate the contributory effects of disruptive changes on travel demand. Authors consider changes in vehicle technology, e.g. automated vehicles, changes in demography, economy and lifestyles. The paper proposes a new FCM approach by introducing expert reasoning and approximate modelling. The proposed approach allows investigating various scenarios for strategic modelling that consider future disruptive changes in travel contexts.

The second set of papers in this issue pertain to techniques to better measure change. To begin, Xiong, Chen, He, Guo and Zhang co-authored the paper, "The analysis of dynamic travel model choices: a heterogeneous hidden Markov approach". This paper tends to overcome the limitations of using static discrete choice models in application of long-term travel demand forecasting. While static discrete choice model can successfully capture crossectional heterogeneity in travel related choice, longitudinal heterogeneity is often overlooked, which might have serious consequences in forecasting changes in travel behaviour in future. Authors found that a hidden Markov modelling (HMM) approach can 
successfully captures longitudinal heterogeneity through their empirical investigation of the Puget Sound Panel Survey data. The proposed approach extends a standard discrete choice model by incorporating state-dependent random error and latent conditioning transition model. Bayesian estimation technique is found to be an easier estimation technique for such type of model and empirical results reveal that the proposed model outperforms its static discrete choice models counterparts.

Paleti, Vovsha, Givon and Birotker co-authored the paper, "Impact of individual daily travel pattern on value of time". They looked at the issue of the variations in subjective value of travel time savings (SVTTS) throughout the day. As opposed to conventional approach of estimating SVTS from univariate mode choice model, it looks at the effects of activity travel patterns and context of travel on SVTS. The study used a GPS assisted travel survey dataset that combines observed travel patterns as well as stated choices information. Empirical model investigates SVTTS in context of a joint mode and time-of-day choice model. The paper clearly proves that travellers perceptions and values of travel time savings vary with time-of-day, different destinations, and activity purposes along with many other factors.

The final paper in this set was co-authored by Wang and Daziano and is entitled, "On the problem of measuring discount rates in intertemporal transportation choices". Authors of this paper tackled a very important issue related to evaluation of transportation planning options, which is how traveller tradeoff alternative choices in intertemporal contexts. Our real life transportation choices involve making choices that have future consequences. For example, the cost of buying a car includes the present cost as well as future maintenance costs. In many such choices, the changes in money value over time are an important factor. Buying a gas guzzler that has a low present cost but which will have higher future maintenance cost versus buying an electric Tesla with a higher current prices but very low future maintenance cost reflect a serious economic decision to make. In such contexts perception of future savings in current decision making is reflected through the assumption of discount rates. Authors review various assumptions of discount rates used in modelling intertemporal choice models. They indentified the approaches of handling discount rates in discrete choice modelling as endogenous and exogenous approaches. The paper also proposes an alternative experimental approach called 'modified reward-over-time' choice experiment that is found very effective in capturing discount rate information in intertemporal choice making involving money and attribute tradeoffs.

Khandker M. Nurul Habib is an Associate Professor of Civil Engineering at the University of Toronto. He works in the area of travel demand modelling, econometric choice modelling, micro-simulation and sustainable transportation planning. He has received numerous awards for his contribution to travel demand modelling and travel behaviour research, which include honorable mention in 2007 Eric I Pass Award from IATBR, 2008 Pyke Johnson Award from TRB and 2013 Minister's Award of Excellence from Alberta Ministry of Transportation.

Stacey G. Bricka (BA, Eckerd College; MA, University of South Florida; PhD, University of Texas at Austin) is Vice President of ETC Institute. Dr. Bricka directs the Travel Behavior Research program and is responsible for identifying, testing and integrating advanced survey methods and data collection technologies for use in transportation research and travel surveys. Her contributions to this journal were performed while she was a Research Scientist with the Texas A\&M Transportation Institute. 